\title{
Formation of Galaxies in the Context of Gravitational Waves and Primordial Black Holes
}

\author{
Shawqi Al Dallal'1, Walid J. Azzam²* \\ ${ }^{1}$ College of Graduate Studies and Research, Ahlia University, Manama, Bahrain \\ ${ }^{2}$ Department of Physics, College of Science, University of Bahrain, Sakhir, Bahrain \\ Email: *wjazzam@gmail.com
}

How to cite this paper: Al Dallal, S. and Azzam, W.J. (2019) Formation of Galaxies in the Context of Gravitational Waves and Primordial Black Holes. Journal of Modern Physics, 10, 214-224.

https://doi.org/10.4236/jmp.2019.103016

Received: January 23, 2019

Accepted: March 1, 2019

Published: March 4, 2019

Copyright () 2019 by author(s) and Scientific Research Publishing Inc. This work is licensed under the Creative Commons Attribution International License (CC BY 4.0).

http://creativecommons.org/licenses/by/4.0/

\section{c) (i) Open Access}

\begin{abstract}
The recent discovery of gravitational waves has revolutionized our understanding of many aspects regarding how the universe works. The formation of galaxies stands as one of the most challenging problems in astrophysics. Regardless of how far back we look in the early universe, we keep discovering galaxies with supermassive black holes lurking at their centers. Many models have been proposed to explain the rapid formation of supermassive black holes, including the massive accretion of material, the collapse of type III stars, and the merger of stellar mass black holes. Some of these events give rise to the production of gravitational waves that could be detected by future generations of more sensitive detectors. Alternatively, the existence of these supermassive black holes can be explained in the context of primordial black holes. In this paper we discuss the various models of galaxy formation shedding light on the role that gravitational waves can play to test of the validity of some of these models. We also discuss the prospect of primordial black holes as a seeding constituent for galaxy formation.
\end{abstract}

\section{Keywords}

Gravitational Waves, Galaxy Formation, Primordial Black Holes, Supermassive Black Holes

\section{Introduction}

The formation of galaxies has been the subject of intensive theoretical and observational work during the past few decades. Observational data reveal the existence of supermassive black holes lurking at the centers of galaxies formed at a 
period very close to the recombination era. However, current observations remain incapable of providing a decisive answer as to the origin of these supermassive black holes. Recent development in the detection techniques of gravitational waves may provide a clue to the processes leading to the formation of supermassive black holes at the center of galaxies. Gravitational waves emanating from these processes in the early universe are extremely weak. Nevertheless, future generations of gravitational wave detectors may shed light on the formation of supermassive black holes in the early universe.

The demography of black holes at the center of galaxies is a promising channel for a better understanding of galaxy formation [1]. The Hubble Space Telescope and the Chandra X-ray Observatory detected supermassive black holes with masses in excess of one billion solar masses in quasars at redshifts corresponding to only a few hundred million years after the Big Bang [2]. The existence of supermassive black holes imposes important constraints on the formation mechanism of galaxies [3]. Furthermore, the gas physics involved in the formation of supermassive black holes is not fully understood yet [4]. So far there is no decisive answer concerning the formation of the earliest black holes, primarily because their growth process masks the origin and properties of the initial progenitor. The logical question that might arise is to what extent modern astronomical observations can set the stage for probing the evolutionary phase of galaxy formation. In this paper, we review some theoretical and observational studies of black holes in the early universe, their possible origin, formation, and fate. In the first part we outline the observational evidence connecting the existence of supermassive black holes in the early universe, followed by a brief introduction to the technique employed in the determination of their masses. In the second part, we present the various models for the formation of supermassive black holes in the early universe, including the collapse of population III stars, dynamical instabilities, the collapse of gas due to dynamical instabilities, the collapse of supermassive stars, the dynamical processes in enriched halos, and the formation and fate of primordial black holes.

\section{Masses of Supermassive Black Holes}

The determination of the masses of black holes is an essential requirement in verifying the degree of accuracy of various galactic formation and black hole growth models and the extent of their adherence to observations. Two important approaches are used to estimate the masses of black holes lurking at the centers of galaxies. The first technique is known as reverberation mapping. It involves the measurement of the structure of the broad emission line region (BLR) around a supermassive black hole. The equation describing this process is given by [5]

$$
G M=f R_{B L R}(\Delta V)^{2}
$$

where $G$ is the gravitational constant, $\Delta V$ is the rms velocity of gas moving near the black hole as determined from the Doppler broadening of the gaseous 
emission lines, $R_{B L R}$ is the radius of the broad line region, and $f$ is a form factor that depends on the shape of the BLR. The measurement of $R_{B L R}$ is considered a serious challenge [6], and the measurement of the $f$ factor is also difficult [5].

The other technique to estimate the masses of supermassive black holes is the use of the M-sigma relation. It represents the correlation between the mass of the supermassive black hole and the velocity dispersion of its host galaxy's bulge [7]. It is used to estimate black hole masses in faraway galaxies, and is given by:

$$
\frac{M}{10^{8} M_{o}} \approx A\left[\frac{\sigma}{200 \mathrm{~km} / \mathrm{s}}\right]^{\alpha}
$$

where $A$ is a constant of order 3, $\sigma$ is the stellar velocity dispersion of the galaxy's bulge, and $\alpha$ is a constant of order 5 representing the slope of the $M-\sigma$ relation. Ferrarese and Merritt [8] found $A=3.1$ and $\alpha=4.8 \pm 0.5$. Other studies gave values very close to the above results [9]. The tight nature of the $M$ - $\sigma$ relation suggests that a feedback mechanism is operating between the growth of supermassive black holes and the growth of the galaxy's bulge.

\section{Processes in Black Hole Formation}

In this section we introduce some basic processes leading to the formation of supermassive black holes. Accretion around a black hole is one of the key ingredients of its growth. The second element is the existence of a dark matter halo that forms and grows from primordial density fluctuations characterized by a virial radius, a mass over-density, and a virial temperature. This halo serves as a host for the pre-galactic disk, which usually grows via gas dynamic processes.

\subsection{Accretion around Supermassive Black Holes}

Accretion around supermassive black holes is considered the only mechanism capable of producing the observed luminosities produced by supermassive black holes in quasars [10]. If accretion is an acceptable mechanism for black hole growth, the progenitor remains controversial, with primordial black holes [11], dark stars [12], and collapsing clouds of gas [13] being the main candidates. The existence of an efficient mechanism for transporting angular momentum outward will enable the accretion material to approach a marginally stable orbit. The existence of magnetic fields in the matter flowing into the disk, as well as turbulent motions, is one such mechanism, since it leads to the transfer of angular momentum outward [14]. The Eddington accretion rate is a characteristic scale for accretion, and is given by [15]

$$
\dot{M}_{E}=3 \times 10^{-8} \frac{0.06}{\eta} \frac{M}{M_{O}} .
$$

The total energy released in the disk is equal to the Eddington luminosity

$$
L_{E}=\eta \dot{M} c^{2} \text {. }
$$

This is a critical luminosity for any given mass $M$, beyond which the radiation force overcomes gravity. Luminosities ranging from $10^{42}$ to $10^{48} \mathrm{erg} / \mathrm{s}$ have been 
observed for active galactic nuclei (AGNs), corresponding to black hole masses ranging from $10^{5}$ to $10^{9}$ solar masses [16]. Even though a good agreement has been found between the observation and the theory of the spectral distribution of radiation, these theories are mainly concerned with mass accretion rates and the luminosity of the accretion disk irrespective of the origin of the accreting supermassive black hole.

\subsection{Primordial Dark Matter Halos}

Galaxies are thought to be formed from baryonic matter in dark matter halos born out of small primordial density fluctuations [17]. There are three important parameters that can be inferred from these halos. The first is the virial mass $M_{v i r}$ that can be calculated directly from the virial theorem. The second is the circular velocity $V_{c}$ which can be calculated from the relation

$$
V_{c}=\left(G M_{v i r} / r_{v i r}\right)^{1 / 2}
$$

where $r_{v i r}$ is the virial radius. The third parameter is the virial temperature, which is given by

$$
T_{v i r}=\mu m_{p} V_{c}^{2} / 2 k_{B}
$$

where $m_{p}$ is the proton mass, $\mu$ is the mean molecular weight, and $k_{B}$ is the Boltzmann constant. The gravitational collapse of the baryon component can proceed when the mass of the over dense region reaches the Jeans mass $M_{r}$. At masses in excess of $M_{F}$, baryons are captured and are then shock-heated by the subsequent collapse and virilization of dark matter. Gas dynamics processes predict that low-mass objects are less efficient in dissipating energy and cool rather slowly, whereas more massive objects can cool at a faster rate [18]. The collapsing halos in the early universe exhibit a virial temperature smaller than $10^{4} \mathrm{~K}$ and are referred to as mini-halos. A necessary condition for the gas to cool down and form the first stars is that the halos should rely on the less efficient $\mathrm{H}_{2}$ cooling [17].

\section{Formation of Supermassive Black Holes}

During the past few decades, several models have been proposed to explain the presence of massive black holes (MBHs) at redshifts corresponding to the era when the universe was less than one billion years old. Important questions to answer are when did the seeding black holes at the center of galaxies form and what mechanism was involved in their growth? Several possible formation channels have been investigated to understand the MBH seed, as outlined below.

\subsection{Collapse of Population III Stars}

Population III stars are massive metal-free objects comprising the first generation of stars after the Big Bang. These stars are postulated to have formed in mini-halos with masses of the order $10^{6}$ solar masses and to have collapsed from the highest primordial density field. For $T_{v i r}>10^{3} \mathrm{~K}$, the cooling process is mediated by molecular hydrogen [18]. Atomic hydrogen cooling takes place in the 
larger halos with a total mass of $10^{8}$ solar masses and $T_{v i r} \geq 10^{4}$. Simulation of the collapse of molecular clouds suggests that massive stars with $M>100$ solar masses can form [19]. The fate of population III stars depends primarily on their masses. The collapse of $40-140$ solar masses low metallicity stars is predicted to directly form a black hole. When the mass of the population III star is in the range 140 to 260 solar masses, the fate of the star is determined by the electron-positron pair production instability that leads to supernova explosions. Supernovae predicted by this model for certain ranges of massive stars will release a colossal amount of energy that can be detected by current observatories. No such events have been recorded so far. On the other hand, the above model has large uncertainties concerning the final mass of the population III stars.

\subsection{Gas Dynamic Instabilities}

Metal-free or metal-poor proto-galaxies are efficient nurseries where black holes can for and grow. In these systems, supermassive black holes can also be formed directly out of a dense gas cloud [20]. On the other hand, enriched halos exhibit an efficient cooling process which favors fragmentation and star formation rather than direct black hole formation. In metal-free gas clouds that characterize the very first proto-galaxies, the collapse is expected to occur only in massive halos with virial temperatures $T_{v i r}>10^{4} \mathrm{~K}$, where the formation of molecular hydrogen is inhibited [21]. At these temperatures $\mathrm{H}_{2}$ formation is inhibited and atomic hydrogen becomes an efficient agent for cooling down the tenuous gas until it reaches $4000 \mathrm{~K}$ [17]. At $T_{v i r}>10^{4} \mathrm{~K}$, the line-trapping of Lyman- $\alpha$ photons in isothermally collapsing gas causes the equation of state to stiffen with the consequence that fragmentation becomes harder to achieve provided that the metallicity does not exceed about $10^{-4}$ of the solar metallicity [22]. The dissociation of $\mathrm{H}_{2}$ in these systems is brought about by Lyman- $\alpha$ trapping. In such halos, gas cooling and contraction proceed gradually with no fragmentation until rotational support halts the collapse, which usually occurs before reaching densities that allow the formation of a massive black hole (MBH).

Local, rather than global, instabilities in a self-gravitating galactic disk can be calculated using the Toomre stability parameter formalism. The Toomre parameter $Q$ is defined as

$$
Q=\frac{c_{s} \kappa}{\pi G \Sigma}
$$

where $\Sigma$ is the surface mass density, $c_{s}$ is the speed of sound, and $\kappa=\sqrt{2} V / R$ is the epicyclical frequency, and $V$ is the circular velocity of the disk. Gravitational instabilities occur when $Q$ approaches a critical value $Q_{C}$ Instabilities might lead to mass in-fall rather than fragmentation and star formation, provided that the destabilization of the system is kept below a threshold value. This happens when the inflow rate is below a critical threshold

$$
\dot{M}_{\text {max }}=2 \alpha_{c} c_{s}^{3} / G
$$

where $\alpha_{c}$ is the viscosity parameter. This process continues until the mass accu- 
mulated at the center $\left(M_{a}\right)$ is enough to make the disk marginally stable. The upper limit of the mass that can contribute to $\mathrm{MBH}$ formation is determined by the mass and spin parameter of the halo.

\subsection{Collapse of Supermassive Stars}

Gas dynamical processes can also lead to the formation of supermassive stars (SMS) that may collapse, under certain conditions, to form an MBH. Gas accumulated at the few parsecs around the center of the halo, by processes described in the previous section, can reach $10^{4}$ to $10^{6}$ solar masses. For efficient gas accumulation, an SMS ( $\left.M \approx 5 \times 10^{4} M_{O}\right)$ may form, which eventually collapses to form a black hole [17]. In systems where mass accumulation is fast enough, the outer layers of the SMS are not thermally relaxed during much of the lifetime of a main sequence star [23]. These stars exhibit complex structures with a convective core surrounded by a convectively stable envelope containing most of the star's mass. Hydrogen burning in the core of these stars is relatively low and continues throughout most of its massive stages. When hydrogen is exhausted, the SMS will contract and suffer catastrophic neutrino losses that lead to its collapse to an initial black hole with a mass of a few solar masses that grows subsequently via accretion from the resulting bloated envelope. This object is referred to as a quasistar [24], and it consists of a low-mass central black hole surrounded by a massive radiation-pressure-supported envelope. The black hole grows gradually at the expense of the massive envelope until the resulting $\mathrm{MBH}$ is unveiled.

\subsection{Dynamical Processes in Enriched Halos}

Star formation can proceed in mini-halos characterized by a virial temperature $T_{v i r}<10^{4} \mathrm{~K}$ [21] [25]. The halos will be enriched with metals by the first generation of population III stars, and thus fragmentation and formation of low mass stars will be a natural outcome of this enrichment [26]. This process sets the stage for new horizons of $\mathrm{MBH}$ formation. Stellar dynamical processes may lead to the formation of compact star clusters [10] [27], resulting from collisions. These collisions arise from dynamical interactions and may play a major role in the formation of very massive stars (VMS) leading to the formation of $\mathrm{MBH}$ remnants in the range $10^{2}-10^{4}$ solar masses [28]. In an attempt to reach equilibrium, the compact core cluster initially contracts and then starts to decouple thermally from its outer region. Energy transfer from the central dense core will cause a rapid core collapse [29]. Dynamical friction causes a segregation of more massive stars in the center. If these massive stars remain in the main sequence stage, then a subsystem will be developed and will decouple from the cluster. In this subsystem, star collisions can proceed in a runaway manner eventually leading to the growth of VMSs [30]. The fate of VMSs depends essentially on their metal enrichment. Metal enriched VMSs will lose much of their mass and end their life as less massive objects $\left(\approx 150 M_{O}\right)$ [31]. The final fate is either a 
low-mass black hole or a pair-instability supernova. For low metallicity, VMSs may have a different fate. Stars with masses $\geq 40 M_{O}$ and sub-solar metallicity may collapse directly into a black hole without a supernova explosion.

\subsection{Dark Stars in the Early Universe}

Dark stars are a new line of research that proposes that the first stars in the universe were fueled by dark matter heating rather than by nuclear fusion [32]. Weakly interacting massive particles (WIMPs) are considered among the best dark matter candidates [33]. It is assumed that in the early universe the density of dark matter was sufficiently high to trigger dark matter annihilation [34]. The annihilation products of WIMPs inside a star can be trapped to produce enough energy to heat its core and prevent its collapse. The first stars are postulated to form inside dark matter halos of masses of the order of 106 solar masses [35], with one single star per halo. It is also argued that these stars set the stage for many important processes like reionization, the seeding of supermassive black holes, and the production of heavy elements in subsequent generations of stars. The lightest neutralino is motivated by supersymmetry (SUSY) arguments and is considered the best WIMP candidate in the Minimal Supersymmetric Standard Model [36].

In the dark star model, authors assumed a mass of $100 \mathrm{GeV}$ for the annihilating WIMPS. So far, WIMPs in general and neutralinos in particular have not been detected despite intensive searches over the past few decades. Furthermore, no trace of supersymmetric particles has been found in the Large Hadron Collider (LHC), even though it attains energies of seven tera-electron volts, which is far in excess of the $100 \mathrm{GeV}$ postulated for annihilating DM particles in dark stars.

\section{Primordial Black Holes}

Theoretical studies of the possibility of formation of primordial black holes (PBH) in the early universe date back to the original work of Hawking [37]. He argued that extreme densities and inhomogeneities in the early universe can lead to the local collapse of matter resulting in the formation of black holes. More recently, Choptuik [37] and Kim [38] demonstrated the formation of PBHs in the inflationary era, during which the energy density of the universe experienced a dramatic decrease leading to a cosmological phase transition. Hawking [37] argues that $\mathrm{PBHs}$ formed in a wide spectrum of masses in the early universe ranging from $10^{-5} \mathrm{~g}$, corresponding to the Planck mass, to $10^{17}$ solar masses. His upper limit for mass exceeds, by many orders of magnitude, even the greatest masses of supermassive black holes observed today in galactic centers. On the other hand, the formation of very small black holes may arise either from the softening of the equation of state, phase transitions, or from the collapse of hypothetical cosmic strings.

Over dense regions in the early universe may collapse to a black hole if the 
gravitational attraction overcomes the pressure forces and the velocity of expansion [33]. This condition is fulfilled when the potential energy for self-gravitation

$$
\Omega \sim-\mu^{2} R^{5}
$$

exceeds the kinetic energy of expansion

$$
T \sim-\mu R^{3} \dot{R}^{2}
$$

where $R$ is the radius of a region in the early universe, and $\mu$ is the energy density. The units are such that $G=c=1$. In a $k=0$ Friedman universe the sum of these energies is zero, therefore

$$
\left(\frac{\dot{R}}{R}\right)^{2} \sim \mu .
$$

Furthermore, Hawking assumed that the equation of state relating the pressure $P$ and the energy density $\mu$ has the form $P=\mu / 3$, and that $\mu$ is proportional to $R^{-4}$. Thus,

$$
\mu \sim t^{-2} \text { and } R \propto \sim t^{-1 / 2} .
$$

A necessary condition for the collapse to occur is that the gravitational energy, $\mu$, should exceed the internal energy $U$. Taking $P=\mu / 3$ and $U \sim \mu \sim R^{3}$, the condition for collapse to occur becomes

$$
\mu R^{2}>1
$$

for $P \sim \mu_{\hat{o}} \log \mu / \mu_{o}$, and $U \sim \mu_{o} R^{3} \log \mu / \mu_{o}$, the condition for collapse reduces to

$$
\mu R^{2}>\frac{\mu}{\mu_{o}} \log \frac{\mu}{\mu_{o}} .
$$

Once a black hole is formed, it will grow by accreting nearby matter. The rate of accretion was calculated by Zeldovitch and Navikov [39]

$$
\frac{\mathrm{d} M}{\mathrm{~d} t} \sim \mu \sim R_{g}^{2} \sim \mu \sim M^{2}
$$

where $\mu$ here is the density of the background universe. But since $\mu=t^{-2}$ (see above), then

$$
M \sim \frac{t}{1+\frac{t}{t_{o}}\left(\frac{t_{o}}{M_{o}}-1\right)}
$$

where $M_{o}$ is the initial mass of the black hole and $t_{o}$ is the time of its formation. Thus, if $M_{o}$ is small compared to $t_{o}$, that is, if the black hole is small compared to the particle horizon, then $M-M_{o}$ remains small and there would be almost no accretion. However, if $M_{o}$ is of the same order as $t_{o}$, then the Zeldovitch-Navikov argument leads to $M \sim t_{o}$. In this case, the accretion would cause the black hole to grow at the same rate as the particle horizon, producing black holes of the order of the Hubble radius if the growth continued to the present time, or it would reach a mass of $10^{15}$ to $10^{17}$ solar masses if the growth 
was at the same rate as that of the particle horizon.

\section{Gravitational Waves from the Relic of Galaxy Formation}

Gravitational waves may be produced extensively during the initial phase of galaxy formation in the early universe. Several models have been proposed to explain the formation of massive black holes at the center of galaxies at redshifts corresponding to the era when the universe was less than one billion years old. Masses of black holes at the center of faraway galaxies can be estimated using the M-sigma relation [8]. An important question to answer is at what epoch the seeding black holes at the center of galaxies were formed, and what are the possible mechanisms involved in their growth. Many hypotheses have been advanced to elucidate the origin of the formation of massive or supermassive black holes at the center of galaxies. As mentioned earlier, several possible formation channels have been proposed for the formation of massive black holes seed [40], collapse of supermassive stars [17], merger of small and intermediate mass black holes. The collision of massive or supermassive black holes at the center of these galaxies will produce energetic GWs that may lie within a sphere of radius again corresponding to the sensitivity of the actual or future GWs detectors [37]. As the sensitivity of gravitational wave detectors improves, they will be capable of detecting GW events at redshifts beyond the era of star formation, and it is highly probable that these detections will provide evidence to support that primordial black holes are involved [41] [42].

\section{Conclusion}

The formation of supermassive black holes at the centers of galaxies in the early universe is one of the most challenging problems in astrophysics. In this paper we introduced the various models of supermassive black hole formation and we compared these models with observational findings. This approach reveals a gap in our understanding of the most efficient processes leading to the formation of supermassive black holes. Gravitational waves may be associated with the formation of supermassive black holes that can be detected by future generations of gravitational waves detectors. Alternatively, primordial black holes could be considered as a good candidate for seeding new born galaxies in the very early universe and in an era when no supermassive black holes could have had the time to form. An analysis of the cosmic background radiation may give a clue about the existence of primordial black holes. Future missions such as the James Webb Space Telescope will reveal the structure of new born galaxies in the very early universe and will provide a wealth of information regarding the nature of the initial seeding black holes at the centers of galaxies.

\section{Conflicts of Interest}

The authors declare no conflicts of interest regarding the publication of this paper. 


\section{References}

[1] Tremaine, S., et al. (2002) The Astrophysical Journal, 574, 740-753. https://doi.org/10.1086/341002

[2] Barth, A.J., et al. (2003) Astrophysical Journal Letters, 594, L95-L98. https://doi.org/10.1086/378735

[3] Haimian, Z. (2004) The Astrophysical Journal, 613, 36-40. https://doi.org/10.1086/422910

[4] Barakana, R. and Loeb, A. (2001) Physics Reports, 349, 125-238. https://doi.org/10.1016/S0370-1573(01)00019-9

[5] Merritt, D. (2013) Dynamics and Evolution of Galactic Nuclei. Princeton University Press, Princeton, NJ.

[6] Peterson, B.M. and Horne, K. (2006) Reverberation on Mapping of Active Galactic Nuclei. In: Livio, M. and Casertano, S., Eds., Planets to Cosmology. Essential Science in the Final Years of the Hubble Space Telescope, Cambridge University Press, Cambridge, UK.

[7] Merritt, D. (1999) Black Holes and Galaxy Evolution. In: Combes, F., Mamon, G.A. and Charmandaris, V., Eds., Dynamics of Galaxies. From the Early Universe to the Present, Astronomical Society of the Pacific, 221-232.

[8] Ferrarese, F. and Merritt, D. (2000) The Astrophysical Journal, 539, L9-L12. https://doi.org/10.1086/312838

[9] Merritt, D. and Ferrarese, F. (2001) The Astrophysical Journal, 547, 140-145. https://doi.org/10.1086/318372

[10] Schneider, P. (2006) Extragalactic Astronomy and Cosmology: An Introduction. Springer-Verlag, Heidenberg.

[11] Barrau, A. (2000) Astroparticle Physics, 12, 269-275. https://doi.org/10.1016/S0927-6505(99)00103-6

[12] Freese, K., Gondolo, P. and Spolyar, D. (2007) The Effect of Dark Matter on the First Stars: A New Phase of Stellar Evolution. Proceedings of First Stars III, Santa Fe, New Mexico, 16-20 July 2007.

[13] Koushiappas, S.M., Bullock, J.S.B. and Dekel, A. (2004) Monthly Notices of the Royal Astronomical Society, 354, 292-304. https://doi.org/10.1111/j.1365-2966.2004.08190.x

[14] Jovanovic, P. and Popovoc, L.C. (2010) X-Ray Emission from Accretion Disks of AGN. In: Wacher, A.D. and Propst, R.J., Eds., Black Holes and Galaxy Formation.

[15] Shakura, N.I. and Sunyaev, R.A. (1973) Astronomy and Astrophysics, 24, 337-355.

[16] Krolik, J.H. (1999) Active Galactic Nuclei. Princeton University Press, Princeton.

[17] Volonteri, M. (2010) The Astronomy and Astrophysics Review, 18, 279-315. https://doi.org/10.1007/s00159-010-0029-x

[18] Tegmark, M., et al. (1997) The Astrophysical Journal, 474, 1.

[19] Bromm, V., Coppi, P.S. and Larson, R.B. (1999) The Astrophysical Journal Letters, 527, L5-L8. https://doi.org/10.1086/312385

[20] Haehnelt, M.G. and Rees, M.J. (1993) Monthly Notices of the Royal Astronomical Society, 263, 168-178. https://doi.org/10.1093/mnras/263.1.168

[21] Bromm, V. and Loeb, A. (2003) The Astrophysical Journal, 596, 34-46. https://doi.org/10.1086/377529

[22] Santoro, F. and Shull, J.M. (2006) The Astrophysical Journal, 643, 26-37. 
https://doi.org/10.1086/501518

[23] Begelman, M.C. (2009) Monthly Notices of the Royal Astronomical Society, 402, 673-681. https://doi.org/10.1111/j.1365-2966.2009.15916.x

[24] Begelman, M.C., Volonteri, M. and Rees, M.J. (2006) Monthly Notices of the Royal Astronomical Society, 370, 289-298.

[25] Abel, T., Bryan, G.L. and Norman, M.L. (2000) The Astrophysical Journal, 540, 39-44. https://doi.org/10.1086/309295

[26] Omukai, K., Schneider, R. and Haiman, Z. (2008) The Astrophysical Journal, 686, 801-814. https://doi.org/10.1086/591636

[27] Clark, P.C., Glover, S.C.O. and Klessen, R.S. (2008) The Astrophysical Journal, 672, 757-764. https://doi.org/10.1086/524187

[28] Devecchi, B. and Volonteri, M. (2009) The Astrophysical Journal, 694, 302-313. https://doi.org/10.1088/0004-637X/694/1/302

[29] Spitzer, L. (1987) Dynamical Evolution of Globular Clusters. Princeton University Press, Princeton.

[30] Portegies, Z.S.F., et al. (1999) Astronomy \& Astrophysics, 348, 117-126.

[31] Gaburov, E., Lombardi, J. and Portegies, Z.S. (2009) Monthly Notices of the Royal Astronomical Society, 402, 105-126.

[32] Spolyar, D., Freese, K. and Gondolo, P. (2008) Physical Review Letters, 100, Article ID: 051101.

[33] Feng, J.L. (2010) Annual Review of Astronomy and Astrophysics, 48, 495-545. https://doi.org/10.1146/annurev-astro-082708-101659

[34] Krauss, L., et al. (1985) The Astrophysical Journal, 299, 1001. https://doi.org/10.1086/163767

[35] Freese, K., et al. (2008) Dark Stars: The First Stars in the Universe May Be Powered by Dark Matter Heating. arXiv:0812.4844v1

[36] Primack, J., Seckel, D. and Sadoulet, B. (1988) Annual Review of Nuclear and Particle Science, 38, 751-807. https://doi.org/10.1146/annurev.ns.38.120188.003535

[37] Hawking, S.W. (1971) Monthly Notices of the Royal Astronomical Society, 152, 75. https://doi.org/10.1093/mnras/152.1.75

[38] Kim, H.I. (2000) Physical Review D, 62, Article ID: 063504. https://doi.org/10.1103/PhysRevD.62.063504

[39] Zel'dovich, Y. and Navikov, I.D. (1971) Relativistic Astrophysics. Vol. 1: Stars and Relativity. University of Chicago Press, Chicago.

[40] Haehnelt, M.G. and Rees, M.J. (1993) Monthly Notices of the Royal Astronomical Society, 263, 168-178. https://doi.org/10.1093/mnras/263.1.168

[41] Choptuik, M.W. (1993) Physical Review Letters, 70, 9-12. https://doi.org/10.1103/PhysRevLett.70.9

[42] Koushiappas, S.M. and Loeb, A. (2017) Physical Review Letters, 119, Article ID: 221104. 